\title{
THE MASS COMPOSITION OF ULTRA HIGH ENERGY COSMIC RAYS
}

\author{
Aurelio F. Grillo* \\ INFN - Laboratori Nazionali del Gran Sasso, SS 17 bis, 67100 Assergi (AQ), Italy \\ * corresponding author: grillo@lngs.infn.it
}

\begin{abstract}
The status of the Mass Composition measurements of Ultra High Energy Cosmic Rays is presented, with emphasis on the results from the Fluorescence Detector of the Pierre Auger Observatory. Possible consequences of the present measurements are discussed, both on the particle physics and astrophysics aspects.
\end{abstract}

\section{INTRODUCTION}

At the highest energies $\left(\log _{10} E>18.5\right)$ Ultra High Energy Cosmic Rays (UHECRs) are very likely of extra-galactic origin. Measurements of the moments of their mass distribution when they hit the Earth's atmosphere are likely to give important clues on their sources, propagation and interaction at (center of mass) energies that are around $100 \mathrm{TeV}$. The zeroth moment (all particle spectrum) by definition does not explicitly distinguish between different nuclear components, although its interpretation can be easily connected to those (see the report by R. Aloisio at this conference). Higher moments are starting to discriminate between different hypotheses, although of course they are more and more affected by statistical and systematic errors.

The most used shower observables for studying the composition of Ultra High Energy Cosmic Rays (UHECR) are the mean value of the depth of shower maximum, $\left\langle X_{\max }\right\rangle$, and its dispersion, $\sigma\left(X_{\max }\right)$. Inferring the mass composition from these measurements is subject to some level of uncertainty. This is because their conversion to mass relies on the use of shower codes which include the assumption of a hadronic interaction model. These interaction models [1] have in common the ability to fit lower energy accelerator data. However, they are based on different physical assumptions to extrapolate these low energy interaction properties to higher energies. Consequently they provide different expectations for $\left\langle X_{\max }\right\rangle$ and $\sigma\left(X_{\max }\right)$. In the following we will mainly discuss the different roles of the two observables, $\left\langle X_{\max }\right\rangle$ and $\sigma\left(X_{\max }\right)$, with respect to mass composition.

On the basis of the superposition model $2\left\langle X_{\max }\right\rangle$ is proportional to $\langle\ln A\rangle$ and therefore it actually measures (average) mass composition for both pure and mixed compositions. The behaviour of $\sigma\left(X_{\max }\right)$ is however more complex, and gives indications on mass distributions corresponding to the same $\langle\ln A\rangle$.

\section{General IDEAS}

As observed above $\left\langle X_{\max }\right\rangle$ can be directly connected to the average composition of the nuclear cosmic rays in the beam when they hit the atmosphere (the average being performed within reconstructed energy bins):

$$
\left\langle X_{\max }\right\rangle=X_{0}+X_{1}\langle\ln A\rangle
$$

where the coefficients depend on details of the interaction of the beam with the atmosphere and generally depend logarithmically on its energy.

For a given combination of nuclear species with normalized (generally energy dependent) weights $\left\{w_{i}\right\}^{1}$

$$
\langle\ln A\rangle=\Sigma w_{i}\left(\ln A_{i}\right)
$$

Here we remark that the nuclear weights at the Earth's atmosphere will be in general different from the corresponding fractions at the sources, since, at the energies we are considering, nuclei will suffer photodisintegration

$$
\gamma A \rightarrow(A-\mathrm{n})+\mathrm{n} N
$$

interacting with the universal radiation backgrounds in the extra-galactic space through which they propagate. This implies that even in the extreme case of sources producing pure compositions of nuclei (apart from protons) the detected composition will be in general mixed.

The variance of $X_{\max }$ is

$$
\begin{gathered}
\sigma^{2}\left(X_{\max }\right)=X_{1}^{2} \sigma_{\ln A}^{2}+\left\langle\sigma_{\text {shower }}^{2}\right\rangle \\
\sigma_{\ln A}^{2}=\Sigma w_{i}\left(\ln A_{i}-\langle\ln A\rangle\right)^{2}
\end{gathered}
$$

while

$$
\left\langle\sigma_{\text {shower }}^{2}\right\rangle=\Sigma w_{i} \sigma_{\text {shower }}^{2}(A)
$$

describes the intrinsic fluctuations of $X_{\max }$ for different nuclei, and as such depends on details of the interactions, but generically decreases with increasing $A$, being maximum for protons and minimum for Iron nuclei [3].

Notice that the first factor in Eq. 2 is trivially zero for a pure composition, while the second factor is obviously larger than zero. Equation 11 describes how the measurement fixes the average (logarithmic) mass, but

\footnotetext{
${ }^{1}$ Given the low statistics at highest energies it is customary to group the nuclei into mass groups.
} 


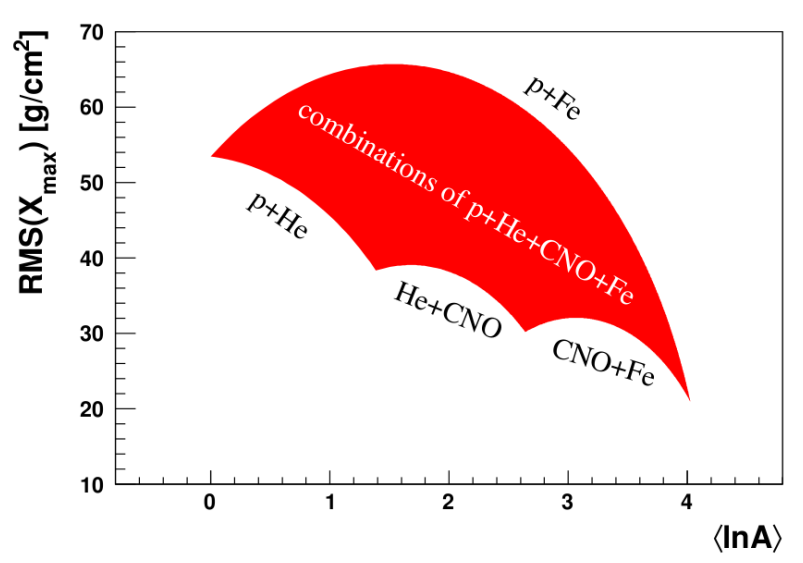

Figure 1 . The relation between $\langle\ln A\rangle$ and $\sigma^{2}\left(X_{\max }\right)$, here for four mass groups (Figure provided by M. Unger).

cannot discriminate the real composition. Equation 2 starts to help in this task because for each average mass there is a spread of corresponding allowed values of the variance - so a given measurement can hopefully exclude possible contributions to the average mass. This is beautifully expressed in a plot, originally proposed by Linsley (Fig. 1) [4. In the original plot the $\sigma_{\ln A}$ was plotted, here we use $\sigma^{2}\left(X_{\max }\right)$, introducing a dependence on the propagation model. It is instructive to elaborate on this plot. The figure describes the possible range of $\sigma^{2}\left(X_{\max }\right)$ for a given value of $\langle\ln A\rangle$. Clearly the cusps correspond to pure mass compositions: here the $\sigma^{2}$ reaches a minimum consistent with a given average mass since the first term in Eq. 2 vanishes. For the same reason the transition from a pure composition to the next one (e.g. from proton to helium) bounds its minimum variation. On the other hand a superposition of the two extreme masses, here proton and iron, gives the largest variance. In fact, for a given $\langle\ln A\rangle$ this combination requires a proton fraction larger than any other combination, and protons give the largest contribution to both terms of Eq. 2.

\section{The Data from the Pierre Auger Observatory}

Figure 2 shows the all-particle spectrum obtained by the Pierre Auger Observatory. The cut-off of the spectrum at high energies has a significance of $\approx 20 \sigma$.

The composition data from the Pierre Auger Observatory discussed below are obtained from hybrid events, which are events detected by the Fluorescence Detector (FD) of the Observatory, with at least a signal in one of the water Cherenkov stations of the Surface Detector (SD) measured in coincidence ${ }^{2}$

Considering the data from December 2004 up to September 2010, after the FD quality cuts [5] 15979 events remain. For the composition analysis additional cuts are used to ensure that no bias

\footnotetext{
${ }^{2}$ The data are those described in Ref. [5] as updated in [6].
}

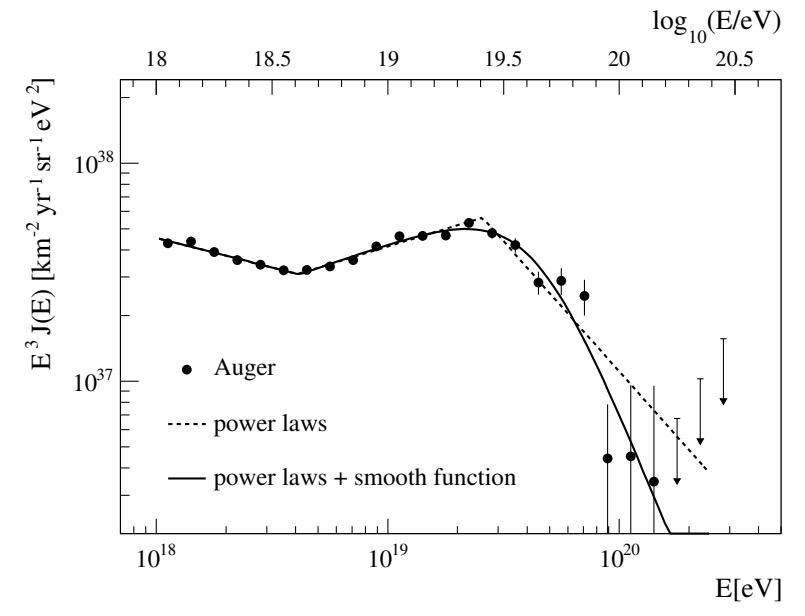

Figure 2. The Pierre Auger Observatory All-particle spectrum. See [7] for details.

with respect to the cosmic ray composition is introduced in the data sample. Specifically, it is required that the trigger probability of the SD station be saturated both for proton and iron primaries and only FD reconstructed geometries are kept for which the full range of $X_{\max }$ is observable. After these cuts, 6744 events remain. The systematic uncertainty in the energy reconstruction of the FD events is $22 \%$ The average resolution is $\approx 20 \mathrm{~g} / \mathrm{cm}^{2}$ over the energy range considered. Furthermore, the $\operatorname{RMS}\left(X_{\max }\right)$ has been corrected for the detector resolution. Therefore the Auger data are detector independent and can be directly compared with simulations.

Let us first consider the plot of $\left\langle X_{\max }\right\rangle$ in Fig. 3 Its energy dependence has been fitted with a broken line, showing an increase up to $\log _{10} E \approx 18.4$ up to a value compatible with a pure proton composition, then a much milder increase consistent with a compensation of the logarithmic increase with energy by a (logarithmic) increase of average mass. As is also indicated by the expectations from interaction models the composition appears to become increasingly heavy with energy. We can therefore conclude that $\left\langle X_{\max }\right\rangle$ data are consistent with $\langle\ln A\rangle$ increasing with energy, at least above $\log _{10} E>18.4$.

Let us now discuss the plot of fluctuations, the lower panel of Fig. 3. Let us first note that this plot can lead to misleading interpretation in the way it is customarily presented. The lines of the predictions of interaction models are only for pure compositions. While in the $\left\langle X_{\max }\right\rangle$ plot they have the generic meaning of the possible range of values of $X_{\max }$, this is not true here and, as for instance indicated in Fig. 1, the fluctuations can be larger than those corresponding to the upper lines, i.e. pure proton compositions, while they cannot be smaller than those of pure iron composition. To take an example, if the composition is proton dominated at some energy and then evolves toward larger mass through a proton-iron combination, then in general in some energy range the fluctuations can be larger than those of pure protons. 


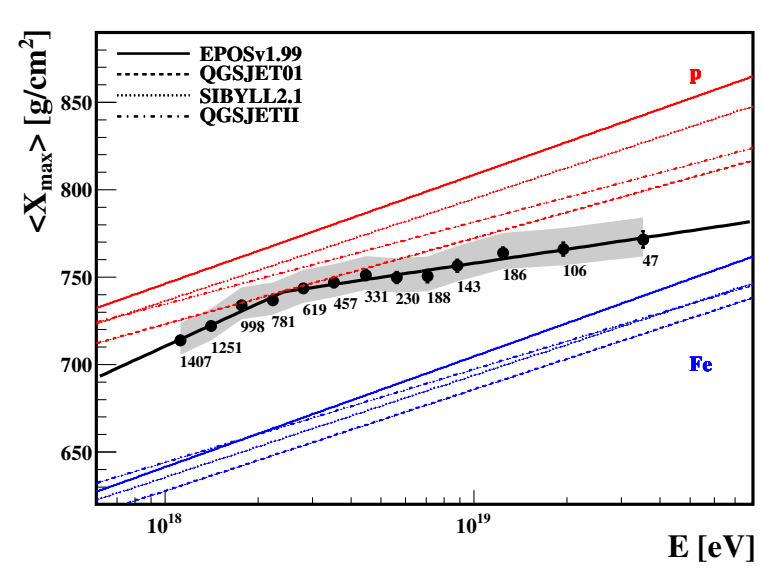

Figure 3. $X_{\max }$ (upper panel) and $\operatorname{RMS}\left(X_{\max }\right)$ compared with predicted values for pure compositions in different interaction models.
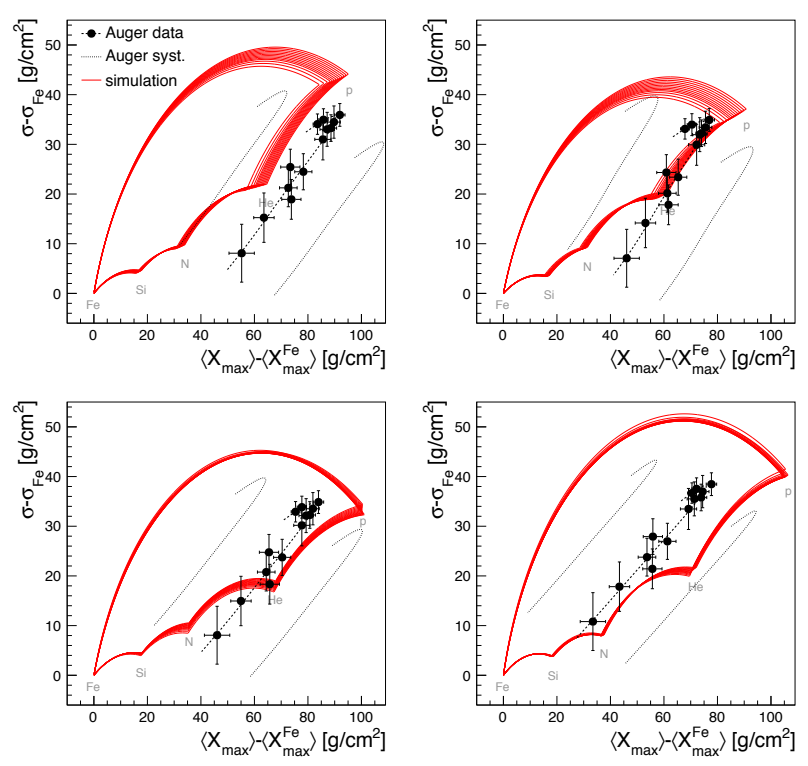

Figure 4. $\sigma\left(X_{\max }\right)$ versus $\left\langle X_{\max }\right\rangle$ of the data, both normalized to iron, compared with predictions of various interaction models: QGSJET01/II (up left and right), SIBYLL2.1 (bottom left) and EPOSv1.99 (bottom right). Energy varies as the dotted line, the highest value being the lower points.

The variance reaches a maximum approximately at the same energy as the break of the slope of $\left\langle X_{\max }\right\rangle$, then it starts to decrease approximately monotonically with energy. Since $\langle\ln A\rangle$ appears to rise monotonically in $\log _{10} E$, and considering Eq. 2 and the behaviour of Fig. 1 it appears that the transition of average mass towards larger values happens consistently through almost pure compositions $\left(\left\langle\sigma_{\ln A}\right\rangle \approx 0\right)$, in particular with few protons at the higher energies.

In [8] a thorough discussion is presented of mass composition measurements in cosmic ray experiments. In particular a discussion of the combined $\left\langle X_{\max }\right\rangle$ versus $\sigma\left(X_{\max }\right)$ of the Auger data is analyzed with the help of a series of plots similar to Fig. 1 for each interaction model considered and for five mass groups

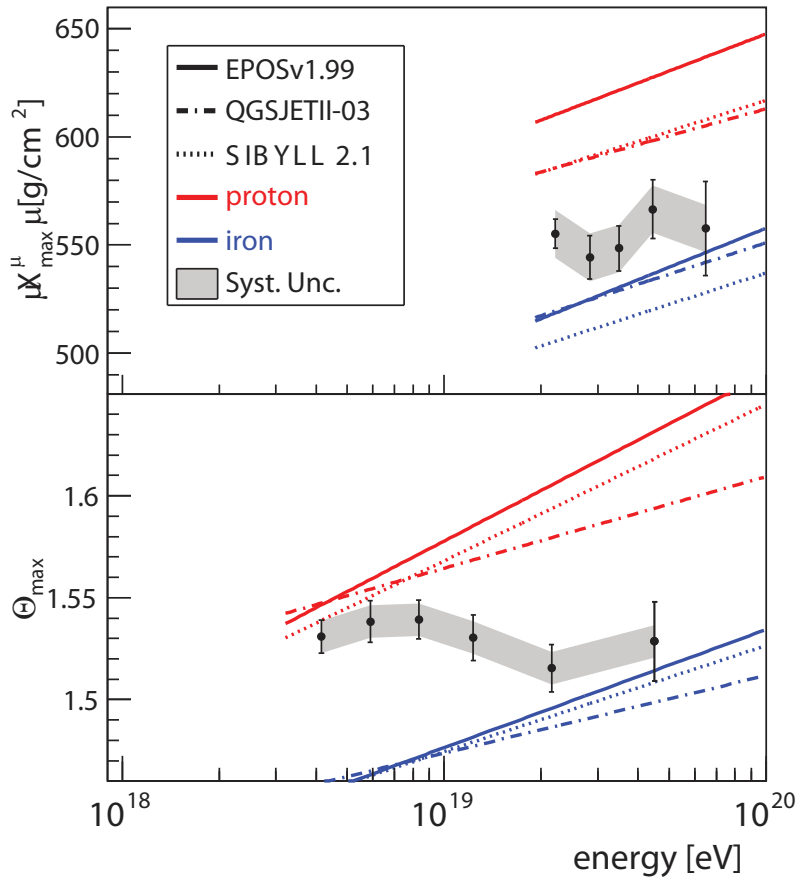

FiguRE 5. Two composition sensitive observables from SD, compared with expectations from interaction models (for further details see [9]).

(proton, helium, CNO, silicon, iron). The main difference from Fig. 1 lies in the use of the experimental observables both for the simulations and for the data. From these plots it appears that the central values of the experimental data (within statistical errors only) have a varying, but generically large, tension towards all the interaction models apart from EPOS, especially at the highest energies. Also, these plots confirm the general idea that the transition to larger masses can be better described by the dominance of different mass groups in different energy intervals, with small mixing between the groups, and in particular little admixture of protons. However, taking into account systematics considerably weakens these conclusions.

The Fluorescence Detector of the Pierre Auger Observatory has only a limited duty cycle, while the Surface Detector is continuously active, therefore composition-related observables connected to SD, although of less direct interpretation, are a valuable complement for direct longitudinal shower developement measurements. Fig. 5 shows two such measurements, relating to muons (and electrons) in Auger showers. This data confirm the FD measurements, especially when systematics is taken into account $[9$.

\section{Discussion}

Although a full analysis of the Auger composition data is not yet available, some tentative conclusions can be drawn. But before discussing them, it is important to relate the behaviour of the composition to the data of other large experiments, HiRes [10, Telescope Array [11] and Yakutsk [12. The former is no longer in operation. These experiments claim a composition 


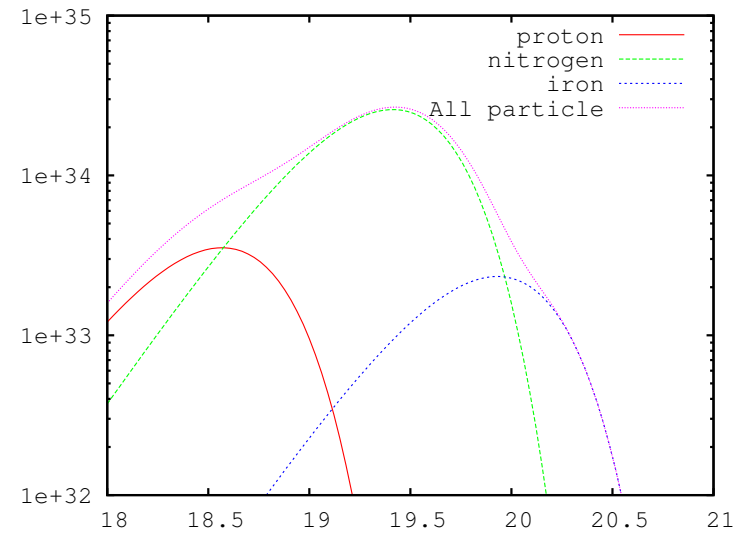

Figure 6. Partial spectra (multiplied by $E^{3}$, in arbitrary units) of a mixture of mass groups that would qualitatively reproduce both spectrum and composition data.

compatible with pure proton. However their dataset is substantially smaller than that of Auger, and their data are also compatible with Auger data. Moreover, with the cuts described above the Auger data (and, although with a different strategy, those from Yakustsk) are free from detector biases and therefore can be directly compared with simulations from interaction models, while for HiRes and TA the detector biases have been applied to the simulations. This makes a direct comparison of the data difficult.

It should also be stressed that moving the data within the relatively large band of systematics, especially for the second moment (the variance of $X_{\max }$ ), can greatly influence the conclusions, as can be seen in Fig. 4 阝

Finally, we are implicitely assuming that at least some of the interaction models used are correct at these energies, which in the center of mass are approximately two orders of magnitude larger than those in LHC. A change in proton (and nuclei) interactions in these range would have profound consequences on the interpretation of the experimental data.

Coming back to the data we have seen that the behaviour of $\operatorname{RMS}\left(X_{\max }\right)$ seems to suggest an evolution of the mass composition toward larger values with little mixing between mass groups. In other words the transition towards large masses in energy is possibly happening through the dominance of a single mass group, as is sketched in Fig. 6 for three mass groups (proton, nitrogen and iron). Although this figure has to be seen as a guide to the eyes, it is clear that a similar behaviour could apply for $\log _{10} E>18.4$ given that measurements suggest an increasing $\langle\ln A\rangle$ plus a decreasing variance, remembering that one expects a decrease of $\left\langle\sigma_{\text {shower }}\right\rangle$ with $A$.

Note however that these are the spectra at Earth.

\footnotetext{
${ }^{3}$ However, as is clear from the figures, moving the data towards lower values of fluctuations is inconsistent with any interaction model and would imply profound consequences from a particle/nuclear physics point of view.
}

Nuclei generally interact with universal radiation backgrounds (both CMB and EBL) in their travel in the extra-galactic space, and suffer photodisintegration in wich the original $A$ decreases. If one assumes the simplest model of (extra-galactic) sources of UHECRs at these energies: uniformily distributed, with a universal power law spectrum and charge-dependent maximum energy, then it appears that very peculiar conditions must be fulfilled in order to reproduce the experimental moments of the mass distribution. In fact, to avoid overpopulating the partial spectra with species produced in the propagation in the extragalactic space (particularly protons) it appears that a low cut-off energy is needed at the source. This in turn implies very flat spectra (i.e. differential slope $<2$ ) to reproduce the observed data. In this case the observed high energy cut-off of the all-particle spectrum would be a feature of the sources, not of the propagation. These features appear to be at odds with generally accepted ideas of acceleration of UHECRs, but of course this source model can be oversimplified. For instance the spectrum might be dominated by only a few (maybe peculiar) nearby sources (see e.g. [13). And, of course, the interaction models used to describe the data might be inadequate at these energies. This has been advocated in [14].

In conclusion, a combined, full analysis of UHECR data (spectrum, composition and possibly anisotropy) is needed to get hints of their provenience. Such full analysis is likely to require a modification of the simplest models, of particle physics and/or of astrophysics, used up to now to describe the data.

\section{ACKNOWLEDGEMENTS}

All of the considerations expressed here benefitted from very fruitful work and discussions within the Pierre Auger Collaboration; they in any case represent my personal point of view. I thank K.-H. Kampert and M. Unger for allowing me to use figures from their paper 8 .

\section{REFERENCES}

[1] For a recent review see e.g. R. Engel, D. Heck and T. Pierog, Annu. Rev. Nucl. Part. Sci., 61, 467 (2011)

[2] See e.g. T.K. Gaisser, Cosmic Rays and Particle Physics, Cambridge 249 University Press, Cambridge, 1990

[3] See e.g. J. Matthews, Astropart. Phys., 22, 387 (2005) and references therein

[4] J. Linsley, Proc. 19th ICRC 6 (1985) 1

[5] J. Abraham et al. (Pierre Auger Collaboration), Phys. Rev. Lett. 104, 091101 (2010)

[6] P. Facal San Luis for the Pierre Auger Collaboration, Proc. 32nd International Cosmic Ray Conference (ICRC 2011), Beijing, China and arXiv:1107.4804v1

[7] F.Salamida (Pierre Auger Collaboration) Proc. 32nd International Cosmic Ray Conference (ICRC 2011), Beijing, China and arXiv:1107.4804v1

[8] K.-H. Kampert and M. Unger, Astropart. Phys. 35, $660(2012)$ 
[9] D. Garcia-Pinto et al. (Pierre Auger Collab.) in Proc. 32nd ICRC (Beijing, China, 2011), arXiv:1107.4804v1

[10] R. Abbasi et al. [HiRes Coll.], Phys. Rev. Lett. 104 161101 (2010)

[11] C. Jui et al. [TA Coll.], Proc. APS DPF Meeting, arXiv:1110.0133
[12] E. Korosteleva et al., Nucl.Phys.Proc.Suppl. 16574 (2007)

[13] A. M. Taylor, M. Ahlers and F. A. Aharonian, arXiv:1107.2055v1

[14] N. Shaham and T. Piran, arXiv:1204.1488v1 282 